\title{
El proyecto de aula en las escuelas de formación de oficiales de la fuerzas militares y de policía: una escuela para pensar.
}

Jairo Andrés Villalba Gómez. ${ }^{1}$

Betty Zarate Cohecha. ${ }^{2}$

Luis Orlando Ruiz Mendoza. ${ }^{3}$

\author{
"Si un día somos llamados a hacer grandes cosas, es por las \\ pequeñas por las que es necesario llegar a ellas."
}

JEAN GUITTON.

\section{Resumen}

El presente artículo caracteriza la importancia de la investigación en la educación basada en prácticas reflexivas, así como los proyectos de aula como sistema de investigación y de construcción de conocimientos; fundamenta su posición teórica desde cuatro puntos de vista: el punto de vista pedagógico y epistemológico, el hermenéutico, desde las TIC, y finalmente, desde el pensamiento crítico, para luego, a nivel praxiológico, caracterizar la articulación de la investigación formativa y los proyectos pedagógicos de aula, en las escuelas de formación de oficiales de las FFMM y de Policía en Colombia.

Palabras clave: proyectos de aula, investigación formativa, escuelas de formación militar, pensamiento crítico.

\footnotetext{
1. Economista - Universidad Santo Tomás, Especialista en Gerencia de Recursos Naturales - Universidad Distrital "Francisco José de Caldas", Candidato a Magíster en Educación - Universidad Santo Tomás. Grupo de Investigación MEMPED Medios y Mediaciones en Educación a Distancia. Profesional Oficial de la Reserva de la Fuerza Aérea Colombiana, Bogotá, D.C. Correo: jairo.villalba@unimilitar.edu.co.

2. Licenciada en Psicología y Pedagogía - Universidad Pedagógica Nacional. Magíster en Educación - Universidad Externado de Colombia, Especialista en Pedagogía y Docencia Universitaria - Universidad La Gran Colombia, Especialista en Estrategias y Tecnologías para el Desarrollo de la Lectura y la Escritura - Universidad La Gran Colombia. Grupo de Investigación MEMPED Medios y Mediaciones en Educación a Distancia. Bogotá, D.C. Correo: betty.zarate@unimilitar.edu.co.

3. Licenciado en Filosofía - Universidad Santo Tomás, Especialización en Educación y Orientación Sexual - Universidad Manuela Beltrán. Grupo de Investigación MEMPED Medios y Mediaciones en Educación a Distancia. Bogotá, D.C. Correo: luis. ruizm@unimilitar.edu.co.
} 


\title{
Classroom projects Army and Police Academies: A school to think
}

\begin{abstract}
This paper characterizes the importance of research reflexive practice-based education, as well as classroom projects as a research and knowledge building systems. Its theoretical position is grounded on four viewpoints: pedagogical, epistemological, hermeneutic and TIC's. Finally, the research in education is addressed from a critical thinking approach; and then in the praxiological level, it characterizes the articulation of formative research, and pedagogicaim classroom projects, in Military and Police Academies in Colombia.
\end{abstract}

Key words: classroom projects, formative research, military academies, critical thinking.

\section{lntroducción}

El presente ensayo surge como resultado del proyecto de investigación "Articulación de los procesos de formación en competencias, "en y para la investigación”. Código DIS - 968, de vigencia 2012, que desde los ámbitos epistemológicos, praxiológicos y pedagógicos, buscó identificar y caracterizar la cultura investigativa en los programas de formación de oficiales en Ciencias Militares, Administración Aeronáutica, Administración Naval y Administración Policial de las Fuerzas Militares en Colombia, el cual fue realizado por el grupo de investigación MEMPED de la Facultad de Estudios a Distancia FAEDIS de la Universidad Militar Nueva Granada.

Este proyecto de investigación, parte inicialmente desde la naturaleza del Proyecto Educativo de las Fuerzas Armadas PEFA, cuyos lineamientos, consolidan cual ha de ser el sentido de la comunidad educativa, de cada uno de sus integrantes e identifica la proyección de las instituciones armadas hacia la visión del militar y del policía del siglo XXI. Del mismo modo, demarca las políticas educativas a todos los niveles del mando y definió los principios filosóficos, antropológicos, pedagógicos y didácticos que inspiran la misión y la visión del Sistema Educativo de las Fuerzas Militares SEFA, con el fin de convertir a las diferentes fuerzas 
en organizaciones de aprendizaje, investigación científica y tecnológica, generadoras de doctrina, ejercitadas en el arte de pensar para el logro de un sólido criterio militar o policial, donde sus integrantes serán profesionales con excelencia en la formación humanística, y ciudadanos ejemplares orientados al servicio de la comunidad.

Es por ello que, cada una de las fuerzas entendió claramente su responsabilidad en el cumplimiento de su misión asignada a través de sus Escuelas orgánicas, asumiendo el direccionamiento estratégico de una visión educativa coherente con los problemas y necesidades de las Fuerzas, bajo la guía permanente de las cinco líneas de acción estratégica emanadas por el Sistema Educativo de las Fuerzas Armadas SEFA: doctrina, excelencia educativa, investigación, desarrollo e innovación, liderazgo y ética, derechos humanos y derecho internacional humanitario.

En esta misma línea, se considera en el (SEFA), que la educación es uno de los pilares fundamentales para la formación del militar moderno. Es por esto, que cada vez se hace más frecuente escuchar discursos sobre la investigación en el aula y sobre el desarrollo de proyectos pedagógicos de aula al interior de las fuerzas.

La investigación es un tema que está de moda, y por estar de moda, supone que todos los responsables de la formación de las futuras generaciones tienen que estar al tanto del mismo y aplicarlo. En este sentido, los actores directos del proceso de aprendizaje tendrán que formarse en investigación, y más aún, las instituciones educativas deben crear un sistema de investigación que permita introducir reformas a los planes de estudio, impulsar el desarrollo de proyectos de «investigación» en diversas áreas del conocimiento, y participar en encuentros de ciencia y tecnología a nivel nacional e internacional.

Por otra parte, la exigencia de la investigación no parte de modas que se impongan; el reto lo genera el contexto mismo, que privilegia el conocimiento por encima de lo que otrora fuera lo primordial. La máxima "information is power" ha adoptado un papel central en la nueva configuración social. Las instituciones educativas, sin importar su carácter y/o denominación, si no quieren desaparecer dentro de la configuración social, tendrán que escuchar los signos de los tiempos y replantear sus prácticas pedagógicas a la luz de las necesidades del entorno.

El reto de hacer investigación, viene siendo afrontado por las instituciones educativas (con mayor énfasis en las escuelas de formación militar y de policía), con la decisión, el valor y el realismo que de ella se exige, pues más que una moda la investigación ha sido y será, en gran medida en los tiempos, la esencia de la formación no sólo intelectual sino también integral del individuo y particularmente en el oficial que allí se forma. 
Si bien la investigación emerge desde el aula, es ahí donde se debe interpelar a todo el ámbito de la comunidad educativa y a todas las instituciones de educación superior en general; ésta permite generar conciencia acerca de ¿Cómo se están adoptando los procesos de enseñanza - aprendizaje?, ¿Cómo es que se están manejando la relación interpersonal entre estudiantes o estudiantes - aprendizaje? ¿Cómo es la participación de todos los actores responsables en el proceso educativo? ¿Cómo están las escuelas de formación de oficiales de las fuerzas militares y de policía impactando el sector barrial o comunal de su localidad? en fin, una serie de posibilidades que permiten hacer de la investigación un "oxígeno" para que la escuela salga de ese "quietismo asignaturista" donde se encuentra sumida.

La importancia de la investigación en la educación basada en prácticas reflexivas, es trascendental en la sociedad del conocimiento, en tanto hace de la escuela y de las Instituciones de Educación Superior, como es el caso de las escuelas de formación militar y de policía en Colombia, una organización inteligente en la que el papel de la ciencia y de la tecnología es la construcción de un conocimiento localmente pertinente y globalmente competitivo.

Luego, los proyectos de aula son un sistema de investigación y de construcción de conocimientos. Surgen del análisis de los problemas del aula o de temas de interés de los alumnos en relación a los contenidos curriculares o problemas de actualidad. Son proyectos elaborados por uno o más docentes y forman parte de la planificación anual del grado o curso.

Los proyectos de aula seleccionan objetivos, contenidos, prevén recursos técnicos y generalmente apuntan a ampliar o complementar algún objetivo institucional o de la planificación del docente, prestando un apoyo en un momento dado y a su vez, tienen la virtud de poderse modificar rápida y ágilmente. Se sostienen sobre tres grandes pilares: el primero corresponde a un proyecto general del currículum, fundamentado por lineamientos del orden institucional; el segundo, corresponde a un proyecto particular, a partir de los centros de interés, y finalmente, el tercero va unido con la planificación del docente.

Existen dos tipos de proyectos de aula: los proyectos de implementación directa y los proyectos de innovación.

Los proyectos de implementación directa se elaboran con el objetivo de cubrir una demanda concreta, y a la vez, ofrecen una solución específica para un problema concreto.

Los proyectos de innovación, implican una estrategia de indagación previa, ya que podrían pensarse como insumos para futuras implementaciones, aportando ideas innovadoras para la transformación, estrategias adecuadas para llevarlos a cabo, así como, sugerencias a cerca de los aspectos o puntos más críticos o conflictivos que dichas metodologías podrían tener. 
1. ¿Por qué y para qué hacer investigación formativa mediante proyectos pedagógicos de aula?

Existen varios argumentos que justifican y motivan la investigación formativa, mediante la ejecución de proyectos pedagógicos de aula. Estos argumentos surgen tanto de la realidad misma del aula y del proceso de enseñanza - aprendizaje, como de la idiosincrasia y el rol de docente en la práctica reflexiva; igualmente surge la comprensión de lo que acontece en las aulas.

Desde el punto de vista pedagógico y epistemológico. Hace referencia al escenario pedagógico y epistemológico en el que se desarrolla tanto la investigación en el aula como el proyecto de aula en sí. Maldonado (2012) manifiesta que ello consiste en dar razón de la postura pedagógica, didáctica y epistémica que enmarca la disciplina y la profesión objeto de formación, de manera endógena y exógena, hasta llegar a lo estrictamente operativo en un proceso de reflexión acción permanente y en un contexto de aprendizajes deseables para el logro de los propósitos de formación.

Esta descripción de los propósitos pedagógicos coadyuvan al desarrollo de la personalidad del educando, el cambio de comportamiento expresado en el intelecto, el movimiento y la responsabilidad, así como el desarrollo de la eficiencia que enfatiza, en esencia, la formación profesional del sujeto en su competencia social.

Continúa diciendo Maldonado, que en un currículo con enfoque de competencias es importante: "Diferenciar qué se entiende por disciplina y que se entiende por profesión: la primera está más ligada con aspectos derivados de la ciencia y la segunda con los desempeños laborales". Esto facilita la identificación de las competencias básicas con las competencias específicas en relación con el ser, el saber y el saber hacer.

En cuanto al saber pedagógico, es innegable que el docente se mueve en el escenario de la pedagogía y de la didáctica y que la construcción de estos conceptos construye a su vez un concepto de docente ligado al dominio disciplinar.

La pedagogía actual insiste con gran urgencia en la "Enseñanza del pensar". Niño (1998) conceptúa que la inteligencia se ve urgida por la realidad misma a marchar en busca de la realidad. Por ello, la intelección racional es "Intelección de búsqueda".

Esta marcha "inquirente" presenta dos vertientes: una, en cuanto es actividad, la otra, en cuanto es intelectiva. La primera marcha intelectiva es pensar, la segunda es razón. Por eso la actividad pensante tiene carácter de "buceo" en la realidad. Pensar es bucear buscando porqués de acuerdo con la espléndida formula kantiana "las cosas dan que pensar". En tal razón, es la realidad la que activa la inteligencia en su indagación de razones. 
Esto permite mostrar cómo la pedagogía circula como una disciplina reconstructiva que recupera el "saber cómo" y lo constituye en "saber qué", mediante el cual se observa la práctica, de acuerdo con las competencias docentes y con el dominio de un saber sobre una disciplina específica.

Desde el punto de vista hermenéutico: el tema de la "interpretación" y la "comprensión" hermenéutica nos invita a considerar una pregunta fundamental, que en su momento Gádamer en su obra Verdad y Método (1977) formuló de la siguiente manera: ¿Qué es lo que realmente (nos) ocurre cuando interpretamos?.

La hermenéutica es la disciplina de la interpretación y trata de comprender textos, lo cual (de manera más amplia) es colocarlos en un contexto respectivo. Con esto el intérprete los entiende, los comprende frente a sus autores, sus contenidos y sus destinatarios, éstos últimos tanto originales como efectivos.

Por su naturaleza misma la hermenéutica es la disciplina de la interpretación, pues ella puede tomarse como arte y como ciencia, "arte y ciencia de interpretar textos". Los textos no son sólo los escritos, sino también los hablados, los actuados y aún de otros tipos; ya que van más allá de la palabra y el enunciado.

Este aspecto es de gran importancia no sólo desde la didáctica y la pedagogía, en relación con el escenario pedagógico del acto educativo, sino que también ayuda a la estructuración y ejecución del proyecto de aula como herramienta y propuesta didáctica de construcción colectiva de conocimiento, fundamentada en la solución de problemas desde los procesos de formación. Al respecto Moreno (2003) citando a Good (1983) resalta el proceso hermenéutico de la interpretación como un enfoque positivo y proactivo de la enseñanza, en el que tanto el docente como el estudiante, participan directamente guiando el aprendizaje mediante preguntas y discusiones.

Como se anotaba anteriormente, en el acto de interpretación confluyen el autor, el lector y el texto; es el terreno en el que se dan cita; el énfasis puede hacerse hacia uno o hacia otro, al extraer del texto el significado. Hay quienes dan prioridad al lector y entonces hay una lectura más bien subjetivista; hay quienes dan prioridad al autor y entonces la lectura es más bien objetivista. Podríamos así hablar de una "intención del texto", pero habría que situarla en el entrecruce del lector con el autor. Así la verdad del texto comprende el significado o la verdad del autor y el significado o la verdad del lector.

Desde el punto de vista de las $\mathrm{TIC}^{4}$. En el mercado comercial abundan diversos aparatos y medios electrónicos como por ejemplo: iPod, iTunes, Laptops, Tablet, Linux, Memorias flash, USB y muchos otros de los que algunos manejamos, entendemos y sabemos su utilidad, pero de otros que no tenemos ni la menor idea. 
Cabe preguntarse entonces ¿Cuál es el uso correcto y oportuno de la tecnología en el aula? evidentemente y para nadie es un secreto que las sociedades, los países y las culturas, están desde hace ya tiempo caminando al ritmo de la tecnología y lo hacen de manera rápida, dinámica y abarcando cada vez más ramas del saber humano. Y más aún, tampoco podemos darnos el lujo de estar totalmente alejados de ellos, cuando las Universidades o las Instituciones de Educación Superior, han optado e institucionalizado el uso de las tecnologías de la información y la comunicación (TIC), como herramienta importante y complementaria en el proceso de enseñanza - aprendizaje en el aula, y en parte de la formación integral que ellas promueven.

Nadie puede desconocer que los jóvenes de hoy, en general, son más flexibles y prácticos en el uso de las nuevas tecnologías y que éstas están presentes en el trabajo, en la calle, en la educación y en los rincones más recónditos de nuestra alma y nuestro cuerpo. Esto compromete a los docentes que deben comprender las reglas de la modernidad en cuanto a "cómo aprenden los jóvenes", cómo incorporar en el currículo las TIC, cómo dialogar con y desde ellas y cómo es que la sociedad se imbrica en ellas.

Al respecto, Maldonado (2012) comenta que dentro del clima de las competencias, y en el marco de los proyectos de aula, hoy se habla en el lenguaje de las TIC de las competencias para investigar. La Educación Superior, en general, ahora pondera entre sus funciones sustantivas o capitales la investigación, y entre los factores de evaluación con fines de acreditación y certificación de sus programas, hacen visible sus resultados en términos cuantitativos o cualitativos, bien sea en docentes investigadores, redes, grupos de investigación, como también en la financiación de infraestructura física y académica.

Por consiguiente, los proyectos pedagógicos de aula deben alinearse acordes con las tendencias educativas para el siglo XXI, en cuanto a temáticas tales como: desarrollo y sociedad del conocimiento; la sociedad del conocimiento y las TIC; la formación y el aprendizaje en la era digital; los nuevos roles del docente y el estudiante en las TIC; los fenómenos y nuevos paradigmas educativos; la alfabetización digital y los nativos digitales; la educación online y los entornos virtuales de aprendizaje, el diseño de programas formativos E-Learning, B-Learning y E-Portafolios.

Desde el punto de vista del pensamiento crítico. Si buscáramos una característica que definiera en pocas palabras el momento en que vivimos, estaríamos de acuerdo en calificarlo como un periodo lleno de constantes cambios culturales, políticos y económicos, que enfrentan continuamente a la educación y los educadores en nuevas situaciones. Este hecho hace necesaria la permanente actitud reflexiva y crítica ante el modelo de sociedad y sus consecuencias culturales: "el pensamiento crítico es importante si las personas funcionan eficazmente en sus medios respectivos". 
En la literatura sobre el tema, no existe unanimidad en una definición de lo que sería pensar críticamente, ni de las condiciones que se deberían dar para juzgar una forma de pensar críticamente. Analizar, evaluar, llegar a conclusiones, emitir juicios, encontrar soluciones a problemas, todas estas operaciones mentales o acciones resultantes de ellas, hacen parte de la naturaleza de la vida diaria de las personas y del pensar en forma crítica.

El pensamiento crítico es una forma de pensar, responsable, relacionada con la capacidad de emitir buenos juicios. Es una "forma de pensar" de quien está genuinamente interesado en obtener conocimiento y en buscar la verdad, y no simplemente en saber argumentar. En este sentido, González (2006) conceptúa que la naturaleza del pensamiento crítico radica en que: "Obtener conocimiento y buscar la verdad, se refiere a la obtención del conocimiento más confiable y a la búsqueda del juicio más razonable, o más sensato, con miras a un propósito”.

Por su parte, Moreno (2003) define el pensamiento crítico como el proceso de estimar conclusiones basándose en la evidencia. Este proceso de establecer conclusiones con evidencia tiene varias formas, por ejemplo: confirmación de conclusiones con hechos, identificación de tendencias y suposiciones implícitas, identificación de información relevante e irrelevante, chequear coherencia.

Afirma Moreno que para ello, son necesarios los procesos básicos cognitivos como parte fundamental del pensamiento, que desarrollan habilidades de pensamiento crítico en actividades de metacognición y metacomprensión, de ciertas actitudes o disposiciones o "hábitos de la mente" como comparar y contrastar, clasificar, inferir, predecir, concluir, figura 1.

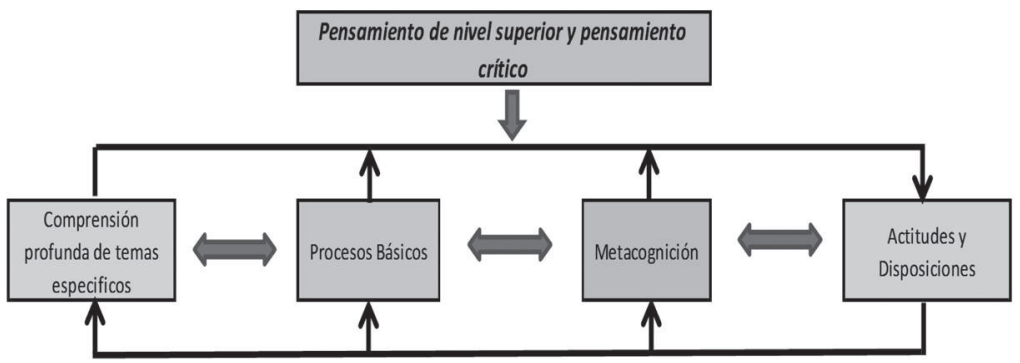

Figura 1. Tomado de: Modelos Educativos Pedagógicos y Didácticos. Vol. I, Pag. 163. Moreno (2003)

De manera más amplia, González (2006) identifica una serie de destrezas intelectuales, capacidades y de disposiciones personales, que debería poseer un individuo para pensar críticamente. Según el autor, "pensar críticamente consiste en un proceso intelectual que, en forma decidida, deliberada y autorregulada, busca llegar a un juicio razonable". 
Este se caracteriza porque es el producto de un esfuerzo de interpretación, análisis, evaluación e inferencia de las evidencias y puede ser explicado o justificado, por consideraciones evidenciables, conceptuales, contextuales y de criterios en las que se fundamenta. Poseer una destreza intelectual, según Gutiérrez: "Es la suficiencia o habilidad para aplicar un cuerpo de conceptos, metodologías o técnicas particulares”.

Estas destrezas intelectuales son necesarias para la interpretación (categorización y descodificación de significados), el análisis (examinar ideas, identificar y analizar argumentos), la evaluación (valorar enunciados y argumentos), la inferencia (examinar evidencias y deducir conclusiones), la explicación (justificar procedimientos y presentar argumentos) y la autorregulación (auto examinarse, autocorregirse, como se evidencia en la figura 2.
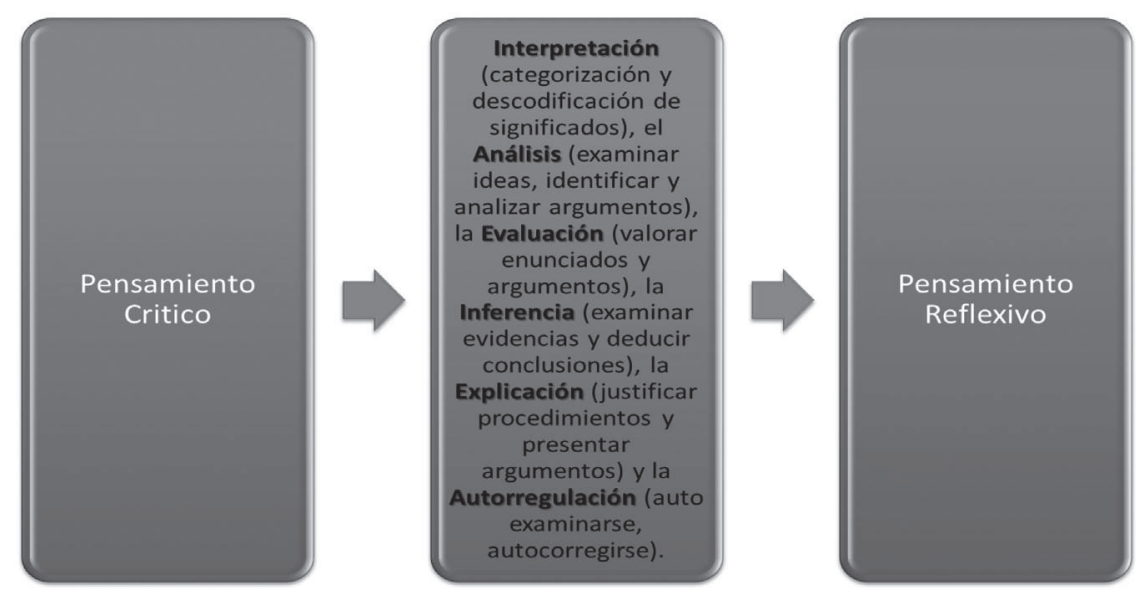

Figura 2. Elementos del pensamiento crítico al pensamiento reflexivo.

Por otro lado, las disposiciones intelectuales son definidas como tendencias adquiridas a pensar en una cierta forma, o siguiendo un cierto patrón, en unas condiciones dadas. La característica más importante de una disposición intelectual es que su ejercicio o utilización se hace en forma reflexiva, es decir, las disposiciones intelectuales no son automáticas.

Peter Facione y otros (2001) identifican y describen cuál debe ser el perfil del pensador crítico ideal: "El pensador crítico ideal, es una persona que es habitualmente inquisitiva; bien formada, que confía en la razón; de mente abierta; flexible; justa cuando se trata de evaluar; honesta cuando confronta sus sesgos personales; prudente al emitir juicios; clara con respecto a los problemas o situaciones que requieren la emisión de un juicio; ordenada cuando se enfrenta a situaciones complejas; razonable en la selección de criterios; enfocada en 
preguntar, indagar, investigar; persistente en la búsqueda de resultados tan precisos como las circunstancias y el problema o la situación lo permitan”.

Finocchiaro (1997) complementa los criterios expuestos por Peter Facione, en cuanto éste agrupa bajo el pensamiento crítico, el razonamiento crítico y la reflexión metodológica, considerando que cada uno de ellos corresponde a una de las dos connotaciones importantes en la crítica: por un lado, el análisis y la evaluación, y por otro lado, la reflexión consciente. Reafirma Finocchiaro, que el razonamiento es la actividad de la mente, que consiste en dar razones para conclusiones, en llegar a conclusiones basándose en razones, o en deducir consecuencias a partir de premisas. Razonamiento es, entonces, una forma especial de pensamiento.

Con respecto a la reflexión metodológica, Finocchiaro la define como el pensamiento encaminado a comprender y evaluar los propósitos, supuestos y procedimientos utilizados en la búsqueda de la verdad o del conocimiento. Esto permite inferir entonces, que el pensamiento crítico es el resultado de la sumatoria entre el razonamiento crítico y la reflexión metodológica.

En este orden de ideas, los proyectos aula como sistema de investigación y de construcción del conocimiento, y como proceso técnico, metodológico y operativo, tienen una función sustantiva y una conexión complementaria directa con los semilleros de investigación, los grupos de investigación y sus correspondientes líneas de investigación institucionales.

2. Cómo se articula la investigación formativa y los proyectos pedagógicos de aula en las escuelas de formación de oficiales de las FFMM y de Policía en Colombia?

Desde el espacio pedagógico, epistemológico, hermenéutico, tecnológico, y propiamente dicho, del pensamiento crítico y reflexivo, las escuelas de formación de oficiales de las FFMM y de Policía en Colombia, a través del tiempo, se han convertido en una auténtica escuela para pensar. Ya en escenarios como el SEFA y el PEFA, a través de la diversidad de ambientes de aprendizaje, se reitera la necesidad de crear cadenas de aprendizaje, para evidenciar la importancia de incluir aspectos, de orden didáctico, pedagógico, y metodológico, que complementen la formación de oficiales, en la construcción del conocimiento, del saber, la planificación del trabajo científico y la formación de proyectos de investigación, que fortalezcan el desarrollo de competencias investigativas.

El SEFA, dentro de sus pilares fundamentales, plantea la necesidad de desarrollar competencias desde el pensamiento analítico, sintético, critico, creativo y sistémico, que potencien la investigación y favorezcan el pensamiento complejo. De la misma manera, el PEFA, reitera los procesos de formación, capacitación, instrucción y entrenamiento desde una perspectiva operativa que 
consolida unas macrocompetencias (ser, saber, saber hacer y saber convivir) a nivel práctico y/o instrumental.

A nivel operativo esas macrocompetencias, están estructuradas en cuatro escenarios: proceso, instrucción, arte y formación, figura 3.

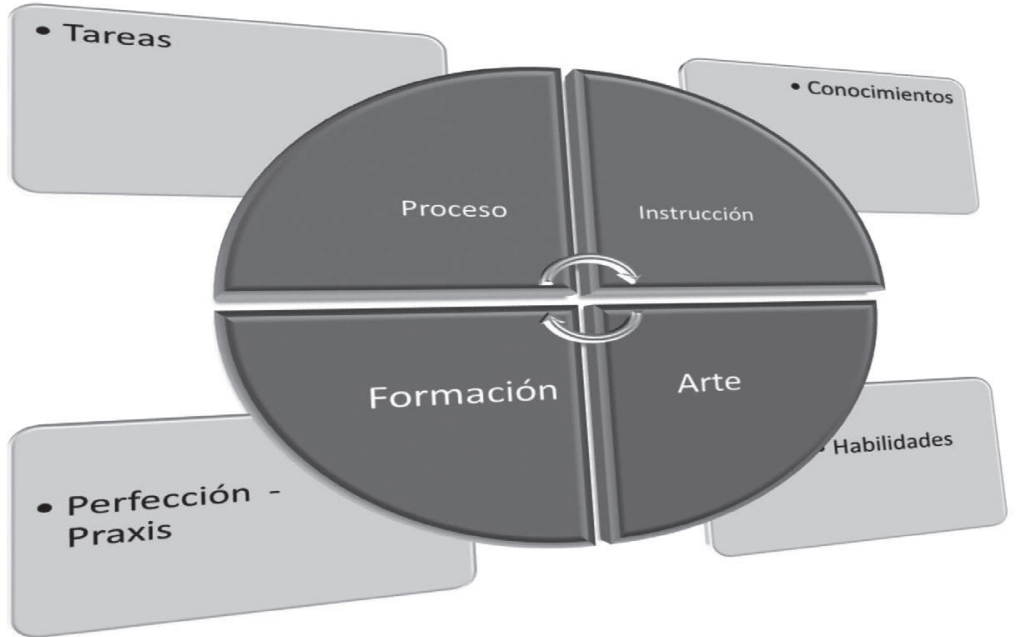

Figura 3. Escenarios de las Macrocompetencias Operativas.

En el plano de los centros de interés de Ovidio Decroly, conviene explicar en primer lugar las características más importantes que definen este modelo de intervención en el aula, entre las que se resalta las facilidades que ofrece al docente y sus alumnos el tratamiento de un conjunto de contenidos que se agrupan en torno a un tema central que da nombre al mismo, elegido en función de las necesidades e intereses básicos de los alumnos.

Sánchez (1995), citando a Decroly, consideraba que las necesidades básicas de todos los seres humanos eran similares y, por lo tanto, los centros de interés inspirados en ellas tendrían una validez general, siendo igualmente útiles para el proceso de construcción del aprendizaje de cualquier grupo de alumnos. Prosigue el autor indicando que la elección de los centros de interés debe hacerse en base a las características de la comunidad educativa a la que pertenecen los alumnos, y por tanto dentro del contexto en el que se desenvuelven habitualmente, adaptando así la intervención docente a la realidad de cada centro educativo. Ello contribuirá a concretar aquellos objetivos que se consideren más importantes para el desarrollo integral de los alumnos.

Estos objetivos, como se ha reiterado anteriormente, se relacionan con las necesidades e intereses básicos de los alumnos en los programas objeto de 
estudio, y por tanto es útil definirlos como centros de interés, entorno a los cuales se van a aglutinar los contenidos más relevantes que serán estudiados por los alumnos.

Los centros de interés son ideas fuerza en torno a las cuales convergen las necesidades fisiológicas, psicológicas y sociales de las personas, de tal modo que se consideran contenidos culturales básicos en los procesos de aprendizaje que deben realizar los alumnos y en este sentido, tienen una mayor estabilidad y permanencia que realiza el equipo docente.

En segundo lugar, los centros de interés concebidos como un conjunto de conocimientos culturales básicos, es preciso que se reflejen en una bitácora o documento de trabajo, que planifica cada etapa educativa por ciclos, a la vez que marcan un cierto estilo pedagógico propio del centro educativo apoyándose en el análisis de la realidad donde éste está ubicado.

En tercer lugar, los centros de interés se ubicarán en los distintos ciclos de la etapa formativa, siguiendo las secuencias definidas por cada una de las áreas que la componen, apoyándose en el orden y las relaciones que se establecen entre los distinto contenidos, garantizando la adecuada progresión y coherencia entre los aprendizajes a lo largo de los distintos ciclos por los que pasa cada grupo de alumnos.

En cuarto lugar, los centros de interés deberán ser planificados por todo el equipo docente que interviene en el ciclo, ya que se considera al ciclo como la unidad de evaluación, y por tanto de la planificación, dentro del organigrama general de la comunidad educativa.

Por último, no representan por tanto programaciones cerradas, ya terminadas y sin posibilidad de modificación, sino que representan una planificación de estos centros de interés posteriormente a cada grupo-clase. Serán, por consiguiente, unos esquemas de relaciones generales de objetivos y contenidos que orientan la evaluación, la distribución de tiempos y espacios, y la selección amplia de materiales, recursos, etc, con el fin de facilitar la actuación de los docentes en las aulas.

En cuanto a los proyectos de trabajo, estos se entienden como el modo de organizar el proceso de enseñanza-aprendizaje abordando el estudio de una situación problemática para los alumnos, que favorece la construcción de respuestas a los interrogantes formulados por éstos. Están relacionados con la realidad y parten al igual de los centros de interés, de los intereses de los alumnos, lo que favorece la motivación y la contextualización de los aprendizajes, a la vez que aumenta la funcionalidad de los mismos, y propicia su aplicación a otras situaciones distintas de las estudiadas en el aula.

Entre sus características más destacadas, los proyectos de trabajo propician una mayor participación de los alumnos en la planificación y desarrollo del 
proceso, creando condiciones más favorables para que se dé una respuesta más amplia y profunda por parte de éstos. Además, se aborda el tratamiento de un mayor número de contenidos que aquellos inicialmente previstos, debido al dinamismo del propio proceso, en el que los alumnos mediante su investigación tienden a relacionar constantemente unos aprendizajes con otros, ampliando así el campo de estudio, que necesita abundante y variado material didáctico, una distribución de espacios y tiempos más flexibles.

En consecuencia, el docente asume un papel más creativo al tener que orientar un proceso rico en situaciones de aprendizaje, que ejerce una constante participación de todos los elementos que intervienen en él (alumno, docente y contenidos), requiriendo un mayor grado de globalidad en su organización.

De igual manera, destaca la importancia de los procedimientos, conceptos y actitudes para facilitar el tratamiento de la información, adquiriendo mayor relevancia las estrategias de aprendizaje, que contribuyen a favorecer la autonomía de los alumnos a través de la reflexión sobre la forma en que construyen sus propios aprendizajes.

Los proyectos de trabajo son distintos a los centros de interés, pero en modo alguno suponen alternativas contrapuestas, ya que estos se planifican dentro del equipo docente con una concepción abierta y a un nivel de generalidad que permite su concreción creativa por el docente en el aula. Suponen además, un modo de organización relacionado directamente con el aula y por tanto con un grupo de alumnos determinados.

Como consecuencia de la planificación de un centro de interés, pueden surgir diversos proyectos de trabajo que guardan relación con éste y a través de los cuales se profundice en determinados aspectos que han despertado el interés de los alumnos y que nos van a permitir continuar abordando los objetivos y contenidos previstos en el currículo.

Como requisitos previos antes de acometer proyectos de trabajo se destaca que los alumnos deben tener adquiridos unos procedimientos y hábitos suficientes para emprender trabajos individuales y de grupo. Para ello es conveniente que los docentes tengan una experiencia suficiente, para desenvolverse en un clima de clase distinto al acostumbrado tradicionalmente.

Es preciso que se cuente con suficiente material para el trabajo de los alumnos, con una organización del aula que facilite la tarea de buscar información y compartirla con los demás, distribuyendo tiempos y espacios que no generen barreras a la actividad delos alumnos.

Por último, es preciso contar con una secuencia general de objetivos y contenidos que oriente el progreso de los aprendizajes de los alumnos que 
El proyecto de aula en las escuelas de formación de oficiales de la fuerzas militares y de policía: Una escuela para pensar

muestren las principales relaciones que se dan entre ellos, para evitar que se produzcan lagunas importantes en el proceso de aprendizaje.

A nivel de las escuelas de formación, objeto de estudio del proyecto de investigación DIS - 968, se pudo observar que la dinámica que evidencia los contextos teóricos anteriores, puntos de vista a nivel pedagógico y epistemológico, hermenéutico, TIC's y del pensamiento crítico, se vienen dinamizando a nivel curricular, a través de la articulación de herramientas para el desarrollo de competencias cuyo objetivo es permitir la ambientalización de los dominios de la investigación formativa (formación en y para la investigación), que como lo explica figura 4, fortalecen la construcción de competencias o habilidades del pensamiento (habilidades cognitivas y lógico formales), para el procesamiento de la información (habilidades en comunicación oral y escrita), así como las habilidades técnicas propias de la disciplina (habilidades praxiológicas).

Puntualmente, y a nivel de resultados específicos del estudio realizado, la Escuela Militar de Cadetes "José María Córdova" - ESMIC, por medio del programa de Ciencias Militares, recorre secuencialmente a actividades de tipo formativo en investigación, con el objeto de confrontar aspectos pedagógicos y didácticos que apoyen la formación de sus cadetes.

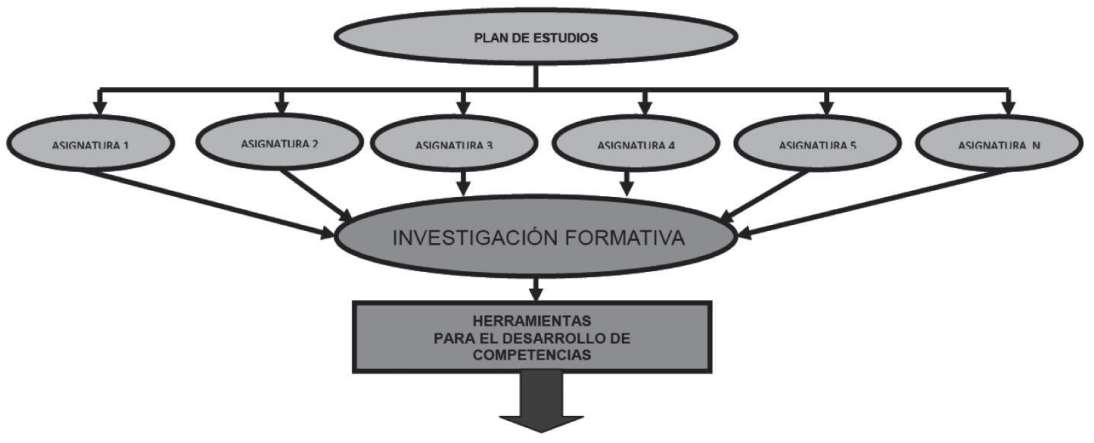

\begin{tabular}{|c|c|c|c|c|c|}
\hline \multirow{2}{*}{ 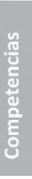 } & \multicolumn{2}{|c|}{ Habilidades de Pensamiento } & \multicolumn{2}{|c|}{$\begin{array}{l}\text { Habilidades para el Procesamiento de } \\
\qquad \text { la Información }\end{array}$} & \multirow{2}{*}{$\begin{array}{c}\text { Habilidades } \\
\text { Técnicas propias } \\
\text { de la Disciplina } \\
\text { Habilidades } \\
\text { PRAXIOLÓGICAS }\end{array}$} \\
\hline & $\begin{array}{l}\text { Habilidades } \\
\text { COGNITIVAS }\end{array}$ & $\begin{array}{l}\text { Habilidades } \\
\text { LOGICO - } \\
\text { FORMALES }\end{array}$ & $\begin{array}{c}\text { Habilidades en } \\
\text { COMUNICACION } \\
\text { ORAL }\end{array}$ & $\begin{array}{c}\text { Habilidades en } \\
\text { COMUNICACION } \\
\text { ESCRITA }\end{array}$ & \\
\hline \multirow{6}{*}{ 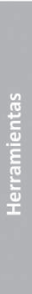 } & Inducción & Análisis Critico & La exposición & El resumen & Tareas \\
\hline & Deducción & $\begin{array}{l}\text { Toma de } \\
\text { Decisiones }\end{array}$ & La conferencia & La reseña & $\begin{array}{l}\text { Talleres y } \\
\text { Ejercicios }\end{array}$ \\
\hline & Inferencia & La Abstracción & La oratoria & La autobiografía & $\begin{array}{l}\text { Trabajos de } \\
\text { campo, Proyectos }\end{array}$ \\
\hline & Análisis & La Comparación & El simposio & El artículo & Laboratorios \\
\hline & Sintesis & La Síntesis & La entrevista & El ensayo & $\begin{array}{l}\text { Seminarios, } \\
\text { Congresos, } \\
\text { Ponencias }\end{array}$ \\
\hline & Otras... & Otras... & Otras... & Otras... & Otras... \\
\hline
\end{tabular}




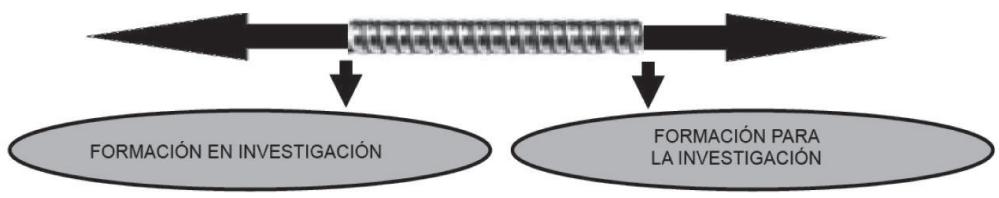

Figura 4. Diagrama de operacionalización de la Investigación Formativa.

Adaptado de Cardona, C. y Villamil, M. (2005)

En el primero y segundo semestre utiliza como mecanismo el Taller Creativo, como medio de identificación de pensamientos e ideas del orden investigativo, así como mecanismos para complementación de competencias en lecto-escritura, secuencialmente. Para el tercer semestre incorpora a sus alumnos al semillero de investigación ayudando en la construcción del Boletín Institucional. Para cuarto y quinto semestre, desarrollan el Seminario Desarrollo Investigativo, escenario en el que, alrededor de un problema de investigación, aplican el conocimiento aprendido y estructuran un plan investigativo de trabajo. En sexto semestre, el alumno recibe un seguimiento académico por medio de tutorías buscando afianzar el objetivo investigativo a desarrollar, en séptimo semestre recibe una complementación académica por medio de un seminario de investigación y sociedad, para finalmente en octavo semestre presentar los resultados investigativos, llamado "trabajo de grado" donde el alumno expone, argumentativamente, su posición investigativa del recorrido investigativo formalizado, a través del tiempo de formación.

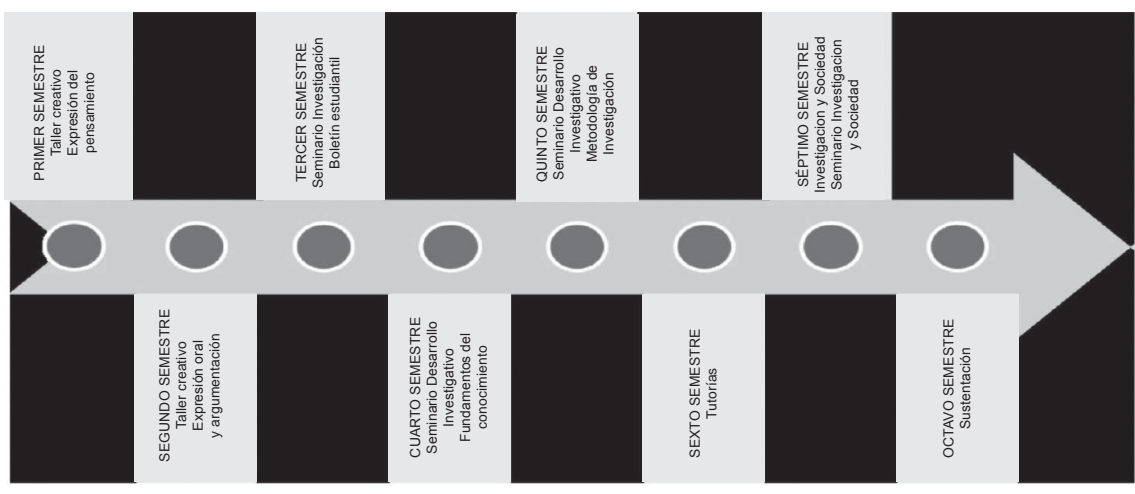

Figura 5. Línea de tiempo de Investigación Formativa. Programa "Ciencias Militares" Tomado de "Guía del trabajo de grado, para el desarrollo de la cultura de la investigación”, ESMIC (2012).

La Escuela Naval de Cadetes “Almirante Padilla” - ENAP, fundamenta su desarrollo investigativo por medio de proyectos de aula, basados principalmente en ejes problémicos, que identifican una estructura metodológica propia para aplicar por año de avance académico. 
Para el primer año, se fortalecen las competencias investigativas en expresión oral y escrita, por medio de la construcción de documentos investigativos tipo ensayos, artículos, redacción de informes, entre otros, los cuales incorporan $\mathrm{y}$ orientan al alumno, en el escenario investigativo. En el segundo año, el alumno recibe orientación metodológica en investigación para identificación de elementos objeto de investigación, reflejados en una propuesta investigativa que se irá consolidando académicamente en los años siguientes. Para el tercer año de escuela, el alumno elabora un anteproyecto de grado, cuyas variables constituyen la identificación metodológica para el desarrollo del problema y sus alternativas de solución, para que finalmente en el cuarto año se consolide el documento investigativo, resultado de la investigación y redacción un artículo publicable, como valor agregado del proceso sistémico de investigación de la escuela.

Tabla 1. Estructura metodológica de los proyectos de aula, Programa "Administración Naval”, ENAP (2012).

\begin{tabular}{|c|c|c|}
\hline CADETES & $\begin{array}{c}\text { TEMA O NÚCLEO } \\
\text { PROBLEMA }\end{array}$ & $\begin{array}{c}\text { ESTRUCTURA } \\
\text { METODOLÓGICA }\end{array}$ \\
\hline Primer Año & Orientación investigativa & $\begin{array}{c}\text { Ensayos, artículos, redacción de } \\
\text { informes, conocimiento de normas } \\
\text { y presentación final de un ensayo } \\
\text { argumentativo de tema libre }\end{array}$ \\
\hline Segundo Año & Emprendimiento & $\begin{array}{c}\text { Elaboración de propuesta de } \\
\text { grado y presentación de ensayo } \\
\text { argumentativo }\end{array}$ \\
\hline Tercer Año & Responsabilidad social \\
empresarial & $\begin{array}{c}\text { Elaboración de ante proyectos de } \\
\text { grado y presentación de un articulo }\end{array}$ \\
\hline Cuarto Año & Tecnología e innovación & $\begin{array}{c}\text { Elaboración del proyecto final y } \\
\text { presentación de un articulo }\end{array}$ \\
\hline
\end{tabular}

La Escuela Militar de Aviación "Marco Fidel Suarez" - EMAVI, por medio del programa Administración Aeronáutica, identifica su actuar investigativo por medio del desarrollo de diversos escenarios en forma simultánea, actividades que se vislumbran a través del proceso de formación de sus alumnos.

En un primer escenario el alumno encontrará, por un lado, los cursos de comprensión lectora, formación para la investigación y metodología de la Investigación para Administración; por otro lado y a nivel de las asignaturas propiamente dichas encontrará: debates, trabajos de curso, relatorías, ensayos, y análisis de casos entre otros, como mecanismos pedagógicos y didácticos que complementan su actuar investigativo.

Un segundo escenario corresponde a la creación y consolidación de Semilleros de Investigación, escenario que permite vincular a los alumnos en el Semillero de investigación en Logística e Industria Aeronáutica (GILOA). 
Finalmente, y en un tercer escenario, los alumnos incorporan su desarrollo investigativo, formalizando las diversas modalidades de trabajo de grado como son: desarrollo del proyecto de grado, diseño y sustentación de un trabajo monográfico modular, realización de pasantías técnicas y/o como auxiliares de investigación en grupos de investigación establecidos.

Tabla 2. Estructura por escenarios investigativos, Programa "Administración Aeronáutica”, EMAVI (2012).

\begin{tabular}{|c|c|}
\hline \multicolumn{2}{|c|}{ Escenario 1} \\
\hline $\begin{array}{l}\text { Articulando la Investigación desde la } \\
\text { Estructura Curricular }\end{array}$ & $\begin{array}{l}\text { Cursos en: } \\
\text { - Comprensión Lectora. } \\
\text { - Formación para la Investigación. } \\
\text { - Metodología de la Investigación para Adminis- } \\
\text { tración. }\end{array}$ \\
\hline $\begin{array}{l}\text { Articulando la Investigación desde } \\
\text { las Asignaturas }\end{array}$ & $\begin{array}{l}\text { - Debates. } \\
\text { - Trabajos de curso. } \\
\text { - Relatorías, ensayos, y análisis de casos. }\end{array}$ \\
\hline \multicolumn{2}{|c|}{ Escenario 2} \\
\hline $\begin{array}{l}\text { Creación y Consolidación de Semilleros de } \\
\text { Investigación }\end{array}$ & $\begin{array}{l}\text { Semillero de investigación en Logística e Indus- } \\
\text { tria Aeronáutica (GILOA). }\end{array}$ \\
\hline \multicolumn{2}{|c|}{ Escenario 3} \\
\hline Modalidades de Trabajo de Grado & $\begin{array}{l}\text { - Proyecto de grado. } \\
\text { - Trabajo monográfico modular. } \\
\text { - Pasantías técnicas. } \\
\text { - Auxiliares de investigación en grupos de } \\
\text { investigación }\end{array}$ \\
\hline
\end{tabular}

La Vicerrectoría de Investigaciones de la Dirección Nacional de Escuelas de la Policía Nacional, desde el programa de Administración Policial, establece puntualmente tres elementos de la investigación formativa que dan evidencia del proceso investigativo dentro del programa.

Un primer elemento corresponde a la presentación formal de resultados investigativos propios de las asignaturas y de los ejes problémicos movibles actualizados a través del tiempo. Estas actividades son de tipo dinámico ya que son elaboradas propiamente por el docente en el desarrollo de sus temáticas de asignatura. Un segundo elemento corresponde al trabajo de grado, constituido como un ejercicio académico y metodológico que engloba un problema de investigación y fundamenta la solución del mismo por medio de una metodología científica. Finalmente, se encuentran los elementos productos de investigación, que a partir de los elementos anteriores, evidencian praxiológicamente los resultados investigativos en la formación de los alumnos de la escuela. 


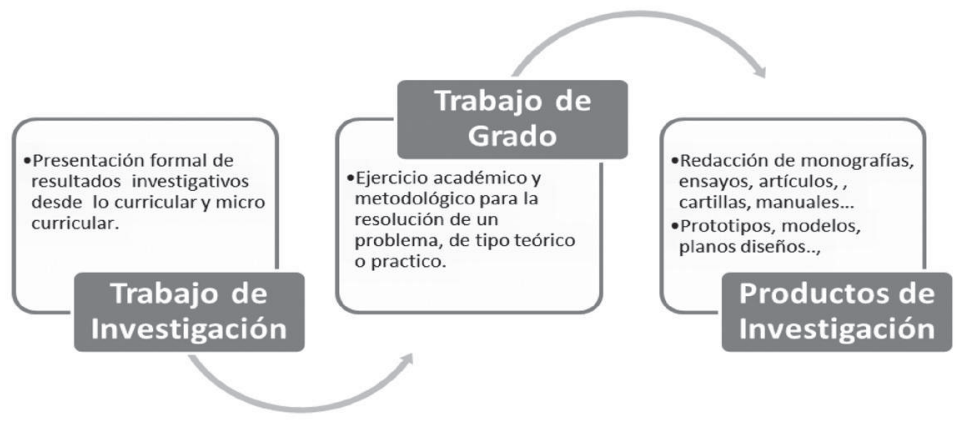

Figura 6. Elementos de investigación, Programa "Administración Policial”, DINAE (2012).

\section{Conclusiones}

Con amplitud temática y de argumentación disciplinar, el tema de proyectos pedagógicos de aula, permite concluir que:

La educación es considerada como uno de los pilares fundamentales para la formación del militar moderno. Ésta, como un proceso integral, permite y facilita la transmisión de conocimientos, valores, costumbres y formas de actuar de hombres y mujeres que hacen parte de la fuerza, con el fin de fortalecer los procesos de formación, capacitación, especialización, instrucción, entrenamiento y reentrenamiento propios del desempeño y dominio militar.

Los proyectos de aula son un sistema de investigación y de construcción de conocimientos. Surgen del análisis de los problemas del aula o de temas de interés de los alumnos en relación a los contenidos curriculares o problemas de actualidad. Son proyectos elaborados por uno o más docentes y forman parte de la planificación anual del grado, curso o periodo académico. Por consiguiente, los proyectos de aula son una estrategia pedagógica para la formación de competencias investigativas.

Generalmente apuntan a ampliar o complementar algún objetivo institucional o de la planificación del docente, prestando un valioso apoyo en un momento dado, y a su vez, tienen la virtud de poderse modificar rápida y ágilmente.

Al relacionar la investigación con los proyectos pedagógicos de aula, es necesario hablar también de los modelos epistémicos del conocimiento científico que como lo explica Barrera (2002), se refieren a la representación del conocimiento o forma significacional que sobre los eventos, las ideas, los hechos de cada cultura o contexto, crea una actividad constante interpretativa. 
Los modelos epistémicos son representaciones conceptuales sobre los cuales se soporta el pensamiento y a partir de los cuales se indaga sobre la realidad. Por ello, el investigador ve lo que ve gracias al modelo que posee. Esto explica por qué la academia (las escuelas de formación de cada fuerza) tiene que hacer un esfuerzo, no solo intelectual sino emocional para romper las barreras que han mantenido modelos pedagógicos transmisores de conocimientos tan arraigados y que se han convertido en barreras al desarrollo del espíritu investigativo en nuestros alumnos.

El papel de la ciencia hermenéutica, como eje de la formación científicohermenéutica del alumno investigador, puede tomarse como el arte y la ciencia de interpretación de textos, hechos y situaciones, que van más allá de la palabra y el enunciado. Este aspecto de gran importancia didáctica y pedagógica, ayuda desde la gestión del conocimiento a la estructuración y ejecución del proyecto de aula como herramienta de construcción del conocimiento.

Para que esto sea posible, es necesario establecer una relación entre modelo pedagógico y el discurso pedagógico oficial o institucional, con el fin de determinar cuál es la clase de conocimiento que ya tiene el alumno y en qué medida este conocimiento le sirve o no para afrontar la tarea de hacer investigación. Aquí es donde llegan nuestros intereses en la epistemología: ¿Cómo se produce conocimiento? ¿Que procedimientos hacen que éste sea legítimo y adquiera tal condición?

En tal sentido, el discurso pedagógico oficial puede entenderse como el instrumento de institucionalización, legitimación y control del modelo pedagógico propio de las escuelas de formación. Para nuestro caso, los centros de interés y los proyectos de trabajo complementan, fortalecen y dinamizan lo proyectos pedagógicos de aula en cuanto que éstos se alimentan de las líneas de investigación disciplinares de dichas escuelas de formación.

Tanto los centros de interés como los proyectos de trabajo, utilizados como herramientas de trabajo e intervención en el aula, facilitan la apropiación y construcción del conocimiento, en cuanto conocimiento significativo, que dinamizan la formación investigativa.

La experiencia del trabajo interdisciplinario, multidisciplinario y transdisciplinario en las ciencias, nos ha enseñado que cada área del conocimiento es una pluralidad de saberes, ámbitos, enfoques y formas de trabajo diferentes.

De allí la importancia de los aportes de todos los saberes disciplinares que aportan un enriquecedor conjunto de conocimientos y experiencias complementarias que contextualizan, enriquecen, innovan y amplían el ámbito de lo pedagógico, la ciencia y la tecnología, de tal modo que las escuelas de formación de oficiales de la Fuerzas Armadas y de Policía desplieguen toda su capacidad técnica y logística con el fin de innovar las metodologías de enseñanza, vincular la educación al entorno en el diseño y desarrollo del currículo, tendientes a la 
mejora de la calidad del sistema educativo de las Fuerzas Armadas y de Policía como organizaciones eficientes.

\section{Referencias bibliográficas}

Barrera, Marcos Fidel. (2002). Modelos epistémicos. Colección Holos, Magisterio.

Facione, Peter. (2001). Pensamiento crítico: su carácter y medida. Diario de la Lógica informal, Vol. 20 No.1.

Finocchiaro, Maurice, A. (1997). Galileo en el mundo de los sistemas. Universidad de California.

González, José Hipólito. (2006). Discernimiento: evolución del pensamiento crítico en la Educación Superior. Icesi.

Maldonado, García, Miguel A. (2012). Currículo con enfoque de competencias. Ecoe ediciones.

Moreno, Moreno, Heladio. (2003). Modelos educativos, pedagógicos y didácticos. $A B C$ del educador, volumen I, editorial SEM.

Niño, Mesa, Fideligno. (1998). Antropología pedagógica. Colección mesa redonda, Magisterio.

PEFA, Proyecto Educativo de las Fuerzas Armadas (2008).

PESE, Plan Estratégico del Sistema Educativo de las Fuerzas Armadas, (2007-2019).

Sanchez, Iniesta, Tomás. (1995). La construcción del aprendizaje en el aula, Magisterio del Rio de la Plata.

SEFA, Sistema Educativo de las Fuerzas Armadas (2010). 